2012

\title{
Column: The Physics of Digital Information-Part 2
}

Fred Cohen

Follow this and additional works at: https://commons.erau.edu/jdfsl

Part of the Computer Engineering Commons, Computer Law Commons, Electrical and Computer Engineering Commons, Forensic Science and Technology Commons, and the Information Security Commons

\section{Recommended Citation}

Cohen, Fred (2012) "Column: The Physics of Digital Information-Part 2," Journal of Digital Forensics, Security and Law. Vol. 7 : No. 1 , Article 1. DOI: https://doi.org/10.15394/jdfsl.2012.1109 Available at: https://commons.erau.edu/jdfsl/vol7/iss1/1

This Article is brought to you for free and open access by

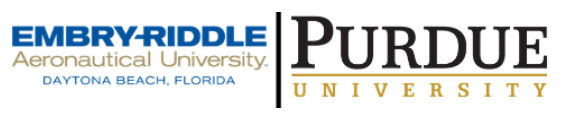
the Journals at Scholarly Commons. It has been accepted for inclusion in Journal of Digital Forensics, Security and Law by an authorized administrator of Scholarly Commons. For more information, please contact commons@erau.edu.

(c)ADFSL 


\section{Column: \\ The Physics of Digital Information-Part $2^{1}$}

\section{Fred Cohen}

In part 1 of this series (Cohen, 2011a), we discussed some of the basics of building a physics of digital information. Assuming, as we have, that science is about causality and that a scientific theory should require that cause $(C)$ produces effect $(\mathrm{E})$ via mechanism $\mathrm{M}$ (written $\mathrm{C} \rightarrow{ }^{\mathrm{M}} \mathrm{E}$ ), we explore that general theory of digital systems from the perspective of attributing effects (i.e., traces of activities in digital systems) to their causes. Full details of the current version of this physics are available online ${ }^{2}$, and in this article, we explore a few more of them.

Previous results questioning consensus around common definitions for the field of digital forensics (Cohen, 2010) have led to additional study suggesting that definitions presented before discussion lead to substantial consensus (Cohen, 2012). Thus each item discussed will start with a loose definition and example.

Definition: A unique history is a single $\mathrm{C} \rightarrow{ }^{\mathrm{M}} \mathrm{E}$ chain that is the only consistent path from the cause to the effect.

For example, suppose we have an imitative copy ${ }^{3}$ of an asserted electronic message sent from one party to another. Given that trace and a set of claims about the computers involved, a unique history would demonstrate that there is one and only one party who could have produced the resulting trace, using one and only one process, at one and only one time, from one and only one place.

\section{Current state does not always imply unique history.}

More generally there are two important rules that are almost always true for the DFE examiner:

\section{Given initial state and inputs, later outputs and states are known.}

\section{Given final state and output, inputs and prior states are not unique.}

Digital systems have a finite number of states (settings of the digital values across all of the stored values in the system). The mechanisms that manipulate digital data are commonly called finite state machines or automata (FSM),

1 This editorial piece is extracted and modified from Cohen (2011c).

2 http://infophys.com/

3 Imitative copy := A reproduction of both the form and content of a record. This is what is typically available and called an "exact", "bit image", or "forensically sound", copy in digital forensics. See Cohen (2011b). 
often detailed in terms of Moore machines (Moore, 1956) or Mealy machines (Mealy, 1955). In such machines, current state and input lead to the next state and output of the machine in a unique way. That is, given the initial state and sequence of inputs, the final state and sequence of outputs are uniquely determined. Thus time transforms the artifice as it moves forward. But in digital forensics, we generally don't start with causes and try to predict effects. Rather, we start with effects and seek to identify their causes. In modern computers it is almost never possible to "run time backwards" given a set of traces, and identify a unique history that led to the traces found.

Definition: Convergence asserts that, as a mechanism transforms inputs and internal states into outputs and subsequent internal states over time, different inputs produce identical outputs. Divergence asserts the opposite, that for the same input, different outputs are detectable.

For example, if we test rolling a rock down a hill repeatedly and, no matter how tightly we control the process, there are slightly different outcomes each time, this would be divergence. But if we ran an FSM forward again and again with different inputs and initial states each time, and got identical outputs and final states, this would be convergence.

\section{Digital space converges while physical space diverges with time.}

The digital artifice over time is, in general, a many-to-one transform. Furthermore, inverting time in an FSM produces potentially enormous class sets of possible prior states and inputs, and determining them precisely is too complex to be done for nontrivial systems (Backes, Kopf, \& Rybalchenko, 2009). This is at odds with the current model of the natural world, in that physical space is generally believed to have an essentially infinite number of possible states and to increase in entropy over time so that order is always reduced. No matter how tightly we control a physical experiment, there will always be a level of granularity at which outputs are differentiable. The difference between the digital and physical spaces is greatly influenced by the fact that the digital space has only finite granularity in time and space, as was discussed in the first article in this series.

Definition: Equivalent machines are, possibly different machines that, from an external perspective, behave identically with respect to a defined set of external data.

For example, different compilers may transform the same program into different binary executable codes that work slightly differently even though they produce the same outputs from the same inputs.

\section{Many FSMs are equivalent.}

An unlimited number of different FSMs may produce the same output 
sequence from the same or different input sequences. For example, at the level of computer programs in common use, an editor, digital recorder, or user program, may produce the same outputs from different inputs. With incomplete traces, we cannot uniquely determine prior states and inputs. To the extent that traces are more or less complete, we may or may not be able to uniquely determine or bound the set of programs that might have produced the traces. We may not even be able to determine the extent of completeness of traces we have.

Definition: A lossy transform is a mapping from input to output that cannot be reversed to produce a unique input. That is, it is a many to one transform.

For example, JPEG files are often compressed using the JPEG lossy compression algorithm (Hamilton, 1992). The results trade off space for quality.

\section{Hash functions and digital signatures as lossy and thus not unique.}

Any transform that produces output space of a predefined size for an input space of a larger size is lossy and thus not unique. As an example, an MD5 or SHA hash of a file does not uniquely identify that file. There are in fact an unlimited number of other files that would produce that same hash value. Being careful, note that this is not an infinite number of files - only an unlimited number of them. To see this, suppose we generate file after file of length one bit more than the length of the hash. Since the length is one bit more, there are twice as many files of that length than there are hash values. If we create one after another of these files, eventually we will exhaust all of the possible values for the hash function, and as soon as we get to one more unique input file than the number of possible hash value, we are guaranteed that two different input files will have identical hash values. This does not make such hashes useless in digital forensics, but it does mean that they do not uniquely identify an input or certify that a produced file is unaltered from its initial creation.

\section{A summary of properties}

There are many other properties of digital systems and the physics of digital information. A summary extracted from the book chapter identified above is included here to expand thinking about these issues.

\begin{tabular}{|l|l|}
\hline Digital World & Physical World \\
\hline Finite time granularity (the clock) & Infinite time granularity \\
\hline Finite space granularity (the bit) & Infinite space granularity \\
\hline
\end{tabular}




\begin{tabular}{|l|l|}
\hline Digital World & Physical World \\
\hline Observation without alteration & No observation w/out alteration \\
\hline Exact copies, original intact & No exact copy, original changed \\
\hline Theft without direct loss & Theft produces direct loss \\
\hline Finite (fast) rate of movement & No locality (entanglement) \\
\hline An artifice created by people & A reality regardless of people \\
\hline Finite State Machines (FSMs) & Physics and field equations \\
\hline Homing sequences may exist & No perfect repeatability \\
\hline Forward time perfect prediction & Forward time non-unique \\
\hline Backward time non-unique & Backward time unique \\
\hline Digital space converges in time & Physical space diverges in time \\
\hline The results are always bits & The results are always continua \\
\hline Results are always "Exact" & Results never perfectly known \\
\hline Time is a partial ordering & Time is real(location) \\
\hline Errors accumulate & Errors are local \\
\hline Representation limits accuracy & Reality is what it is \\
\hline Precision may exceed accuracy & Precision is potentially infinite \\
\hline Forgery can be perfect & Forgery cannot be perfect \\
\hline DFE is almost always latent & Some evidence is latent \\
\hline DFE is trace but not transfer & Traces comes from transfers \\
\hline DFE is circumstantial & Evidence is circumstantial \\
\hline DFE is hearsay & Evidence is physical \\
\hline
\end{tabular}


Digital World

DFE cannot place a person at a place at a time

DFE can show consistency or inconsistency only

Probability is dubious

Content has information density

Content density variable

Content perfectly compressible

Digital signatures, fingerprints, etc. generated from content

Content meaning is dictated by context

\begin{tabular}{|l|}
\hline \\
Context tends to be global and \\
dramatically changes meaning
\end{tabular}

dramatically changes meaning

FSMs come to a conclusion

Cognitive limits from program

Hardware fault models from computer engineering

Time and space tradeoffs known

Near perfect virtualization and simulation possible

Many nearly or equivalent FSMs

Undecidable problems

Computational complexity limits computations

\section{Physical World}

Evidence may put an individual at a place at a time

Evidence can show more than just consistency

Probability is often usable

No defined density limits

Content density not controlled

No perfect compression

Body (phenome) generated from DNA (genome)

No universal theory of meaning but physicality exists regardless

Context tends to be local and incrementally changes meaning

Eats shoots and leaves

Cognitive limits from physiology

Hardware fault models from physics

Tradeoffs unclear

No virtualization

The uncertainty principal

Nothing known as "unthinkable"

No well understood limits on new ideas 


\begin{tabular}{|l|l|}
\hline Digital World & Physical World \\
\hline Everything is decidable & Many things are not decidable \\
\hline Consistency is guaranteed & Consistency is possible \\
\hline Completeness is guaranteed & Completeness is possible \\
\hline Consistency AND completeness & Consistency OR completeness \\
\hline Time limits on achievable results & Time limits unknown \\
\hline Complexity-based designs & Complexity not determinant \\
\hline Fault tolerance by design & Normally not fault tolerant \\
\hline Accidental assumption violations & Assumptions non-violable \\
\hline Intentional assumption violations & Assumptions non-violable \\
\hline Discontinuous space & Continuous space \\
\hline Discontinuous time & Continuous time \\
\hline $\begin{array}{l}\text { Minor differences amplified near } \\
\text { discontinuities }\end{array}$ & Differences retain fidelity \\
\hline $\begin{array}{l}\text { Major differences suppressed away } \\
\text { from discontinuities }\end{array}$ & Differences retain fidelity \\
\hline $\begin{array}{l}\text { Identical use of an interface may } \\
\text { produce different results }\end{array}$ & $\begin{array}{l}\text { No such thing as identical, each thing } \\
\text { is unique }\end{array}$ \\
\hline Ordering may be reversed & Ordering subject to light time \\
\hline Value sorts may be reversed & Value sorts remain consistent \\
\hline Actuate-sensors loop errors & Interference based errors \\
\hline Sensors/actuators limited in physical & All physical properties present \\
\hline
\end{tabular}

Table 1 - Summary of Information Physics 


\section{A final comment}

There is a lot to learn about the physics of digital information, and from the perspective of digital forensics, this is the sort of knowledge that is increasingly necessary to understanding what you are doing when you undertake to testify about such matters.

I urge you to review the details of the physics in its full richness and with its current limitations, and to draw your own conclusions. Read the chapter cited above, comment on it, prove it is wrong if and where it is, show its limits, and move the field forward.

And I urge you to challenge yourself and others to up your game. In case after case, we encounter self-identified experts who don't understand the basics of how things work and end up testifying with inadequate basis. In many cases their conclusions may be right, but their presentation and the facts they provide may not support them. In other cases, their conclusions are not right at all. At the heart of it all is the lack of attention to the basics of the science that underlies digital forensics. This is a problem we hope to continue to address in this series and this publication.

\section{References}

Backes, M., Kopf, B., \& Rybalchenko, A. (2009). Automatic Discovery of Quantification of Information Leaks. In Proceedings of the 30th IEEE Symposium on Security and Privacy, May 17-20, 2009, Berkeley, CA. Los Alamitos, CA: IEEE.

Cohen, F. (2010). Toward a Science of Digital Forensic Evidence Examination. IFIP TC11.8 International Conference on Digital Forensics, Hong Kong, China, January 4, 2010. In K.-P. Chow and S. Shenoi (eds.), Advances in Digital Forensics VI, Springer, pp. 17-36.

Cohen, F. (2011a). Digital Forensic Evidence Examination. Livermore, CA: ASP Press.

Cohen, F. (2011b). Putting the Science in Digital Forensics. Journal of Digital Forensics, Security and Law, 6(1), 7-14.

Cohen, F. (2011c). The Physics of Digital Information. Journal of Digital Forensics, Security and Law, 6(3), 11-16.

Cohen, F. (2012). Update on the State of the Science of Digital Evidence Examination. Submission to the 2012 Conference of Digital Forensics, Security and Law.

Hamilton, E. (1992, September 1). JPEG File Interchange Format, Version 1.02. Milpitas, CA: C-Cube Microsystems. Retrieved from http://www.jpeg.org/public/jfif.pdf 
Journal of Digital Forensics, Security and Law, Vol. 7(1)

Mealy, G. (1955). A Method for Synthesizing Sequential Circuits. Bell Systems Technical Journal, 34, 1045-1079.

Moore, E.F. (1956). Gedanken experiments on sequential machines. In Automata Studies. Princeton, N. J.: Princeton University Press, pp. 129-153. 